\title{
The regulation of cytokine signaling by retinal determination gene network pathway in cancer
}

This article was published in the following Dove Press journal:

OncoTargets and Therapy

\author{
Xinhua Zheng ${ }^{1,2, *}$ \\ Qian Liu',* \\ Ming $\mathrm{Yi}^{2}$ \\ Shuang Qin ${ }^{2}$ \\ Kongming $\mathrm{Wu}^{2}$
}

'Department of Clinical Medicine, Medical School of Pingdingshan University, Pingdingshan, Henan 467000, China; ${ }^{2}$ Department of Oncology, Tongji Hospital of Tongji Medical College, Huazhong University of Science and Technology, Wuhan 430030, China

*These authors contributed equally to this work
Correspondence: Kongming Wu Department of Oncology, Tongji Hospital of Tongji Medical College, Huazhong University of Science and Technology, No 1095 Jie Fang Avenue, Hankou, Wuhan 430030, China

Email kmwu@tjh.tjmu.edu.cn

\begin{abstract}
Tumor environment plays a pivotal role in determining cancer biology characteristics. Cytokine factors, as a critical component in tumor milieu, execute distinct functions in the process of tumorigenesis and progression via the autocrine or paracrine manner. The retinal determination gene network (RDGN), which mainly comprised DACH, SIX, and EYA family members, is required for the organ development in mammalian species. While the aberrant expression of RDGN is involved in the proliferation, apoptosis, angiogenesis, and metastasis of tumors via interacting with different cytokine-related signals, such as CXCL8, IL-6, TGF- $\beta$, FGF, and VEGF, in a cell- or tissue-dependent manner. Thus, joint detection of this pathway might be used as a potential biomarker for the stratification of target therapy and for the precision prediction of the prognosis of cancer patients.
\end{abstract}

Keywords: DACH1, SIX1, EYA1, cytokine, tumor, proliferation, metastasis

\section{Introduction}

Cytokines, secreted by a wide range of cells, take effect on the cellular biological features and behavior in the organic microenvironment via an autocrine, paracrine, or endocrine manner. ${ }^{1}$ They play vital roles in physiological and pathological condition, especially in cancer. In the process of embryogenesis, cytokines are involved in tissue specification. ${ }^{2,3}$ Cytokines, such as $\mathrm{C}-\mathrm{X}-\mathrm{C}$ and IL family, participate in infection and immune responses by recruiting and activating inflammatory cells. ${ }^{1}$ However, the dysregulation of those cytokines results in diseases ranging from autoimmune disorders to cancers.

Retinal determination gene network (RDGN) was first found in the process of Drosophila eye differentiation, and it determined distinct tissue specificity. ${ }^{4}$ This conserved network consists of a dominant suppressor of ellipse, dachshund (dac/ Dach); a tyrosine phosphatase eyes absent (eya/Eya); the Six family transcription factor (TF) sine oculis (so/Six); and two Pax6-like homeodomain proteins, eyeless (ey) and twin of eyeless (toy)., ${ }^{4,5}$ Among them, ectopic expression of DACH-SIXEYA signal presents a novel tumor signal in a coordinated fashion; for example, the downregulation of DACH is often accompanied with the upregulation of EYA and SIX in tumors. ${ }^{6}$

It has been showed that RDGN regulates normal or pathological function in a tissue-dependent manner via interacting with various cytokines. ${ }^{7}$ As the major component, DACH-SIX-EYA signaling regulates the expression and secretion of specific cytokines to affect cell behaviors. In this mini-review, we summarized the regulatory effects of RDGN members on cytokine signaling in relation to the initiation and progression of different tumors. 


\section{Overview of the RDGN signaling}

RDGN is a critical signal in tissue specification and organogenesis, the aberrant of this network is related to various diseases ranging from congenital anomaly (development defect) and cancers. ${ }^{8}$ In vertebrate, $D A C H$ gene encodes two chromatin-associated proteins, namely, DACH1 and DACH2. DACH contains the following two conserved domains: an N-terminal Dach and Sno/Ski homolog domain (DS domain) with DNA-binding ability and a C-terminal EYA domain responsible for protein-protein interactions (Figure 1A). $\mathrm{DACH} 1$ is a crucial component in RDGN through regulating expressions of targeted gene by directly binding to specific DNA sequences or interacting with other TFs (c-Jun, Smads, Six, and ER- $\alpha) \cdot{ }^{9-12}$ In the process of organogenesis, DACH1 is responsible for the tissue development. ${ }^{11,13,14}$ While the abnormal expression of DACH1 leads to various diseases, including bilateral cystic renal dysplasia, ${ }^{15}$ chronic kidney disease, ${ }^{16}$ familial young-onset diabetes, prediabetes, and cardiovascular diseases. ${ }^{17}$ Besides, DACH1 inhibits aldosterone secretion in zona glomerulosa cells. ${ }^{18}$

EYA family has a conserved transactivation domain and EYA domain with four homolog proteins, namely, EYA1 to EYA4 (Figure 1A). It functions as protein phosphatase and transcriptional co-activator for SIX1. ${ }^{11}$ Dysregulation of this compound would cause a range of syndromes. ${ }^{19-21}$
The phosphatase function of EYA stimulates tumorigenesis and metastasis and reverses the antitumor effect of estrogen receptor $\beta$ (ER $\beta)$ in breast cancer. ${ }^{22,23}$

The vertebrate $S I X$ genes encode six homolog proteins, called SIX1 to SIX6. Members of the SIX gene family are characterized by a divergent DNA-binding homeodomain and an upstream SIX domain, which is involved in determining DNA-binding specificity and mediating proteinprotein interactions (Figure 1A). SIX gene governs the tissue determination and development of many organs, including eye, kidney, and muscle. ${ }^{8,24,25}$ SIX proteins are kept at a low level in mature tissues, which are upregulated in many tumor tissues. ${ }^{26} \mathrm{SIX} 1$ regulates expressions of targeted genes through recognizing and binding to specific DNA sequences with the assistance of other cofactors within promoters. ${ }^{27}$ For instance, DACH1 acts as a corepressor, while EYA is a coactivator for SIX (Figure 1B). ${ }^{11,24}$

RDGN signaling plays a significant role in the initiation and progression of tumors. ${ }^{24,28} \mathrm{In}$ general, DACH1 acts as a tumor suppressor, which inhibits growth and metastasis of breast cancer, lung cancer, kidney, prostate cancer, and so on. ${ }^{24,29}$ By contrast, SIX/EYA complex has the ability to promote tumorigenesis and tumor progression. ${ }^{24,29}$ As a result, downregulated DACH1 accompanied with upregulated SIX/EYA lead to the onset and aggressiveness of malignant tumors.

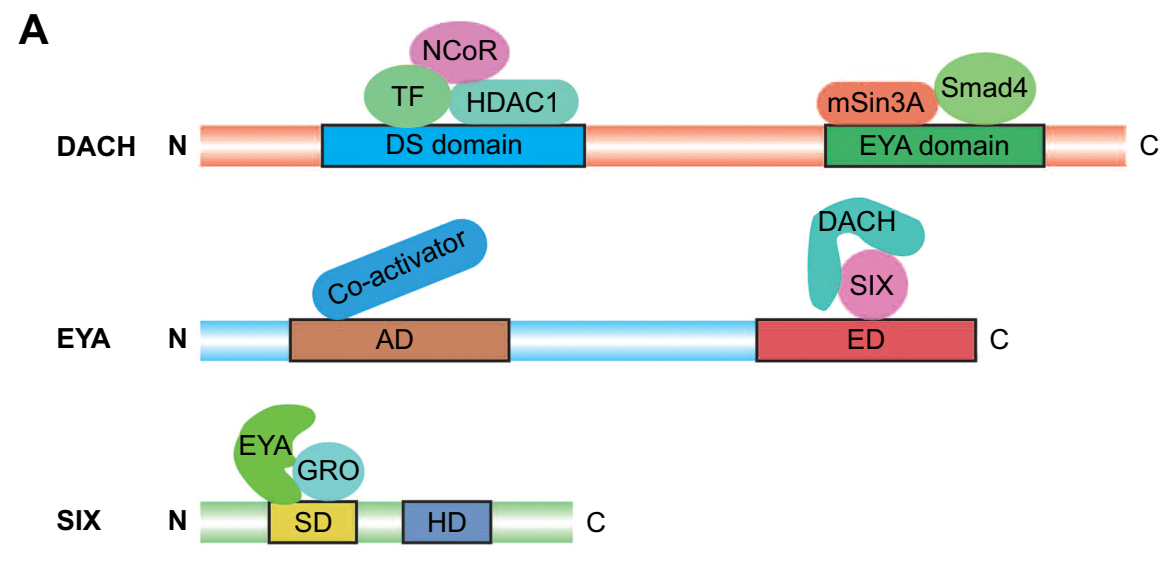

B
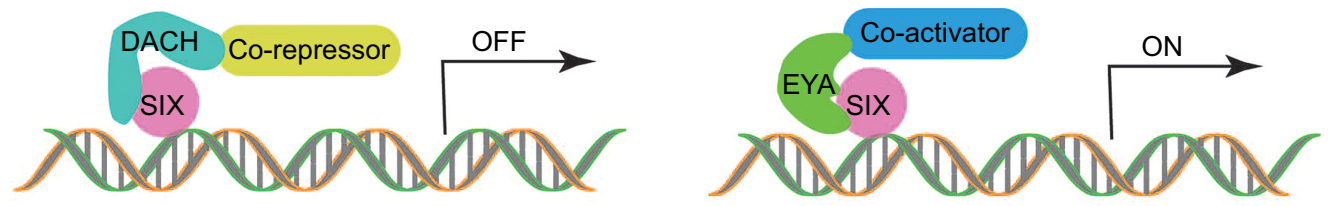

Figure I Schematic structure of Dach, Eya, and Six and their interaction.

Notes: (A) Key structure of Dach, Eya, and Six. (B) Proposed working model of Dach, Eya, and Six to regulate gene expression. AD, transcriptional activation domain; DS domain, Dach and Sno/Ski homolog domain; ED, EYA protein-protein interaction and phosphatase domain; HD, homeobox DNA binding domain; SD, SIX specific protein-protein interaction domain.

Abbreviations: TF, transcription factor; NCoR, nuclear receptor co-repressor. 


\section{Cytokine signaling regulated by RDGN \\ TGF- $\beta$ signaling}

TGF- $\beta$ is a multifunctional cytokine taking diverse effects on epithelial and mesenchymal tissues and regulating cell behaviors, such as cell differentiation, proliferation, and apoptosis. ${ }^{30,31}$ TGF- $\beta$ exerts a Janus-faced role in the process of tumor development depending on the tumor type and stage and genetic/epigenetic changes. ${ }^{32,33}$ Studies showed that TGF- $\beta /$ Smad axis retarded proliferation of epithelial cells in the early stage of tumors, whereas triggered epithelialmesenchymal transition (EMT) procedure and promoted distant metastasis in advanced tumors by stimulating the activation of mesenchymal genes and inhibiting the expression of epithelial genes. ${ }^{34-36}$ Moreover, TGF- $\beta$ is involved in the maintenance of stemness of cancer stem cells (CSCs) in scirrhous gastric cancer and breast cancer. ${ }^{37,38}$

RDGN regulates the tumor process by interplaying with TGF- $\beta$ signaling (Figure 2). On one hand, DACH1 in association with the nuclear receptor co-repressor (NCoR) was shown to block TGF- $\beta$ signaling by binding to Smad4, which in turn restrained TGF- $\beta$ /Smad-induced EMT in breast cancer and gastric cancer (Table 1). ${ }^{39,40}$ Meanwhile, DACH1 shared structural homology to Ski/Sno oncogenes, which
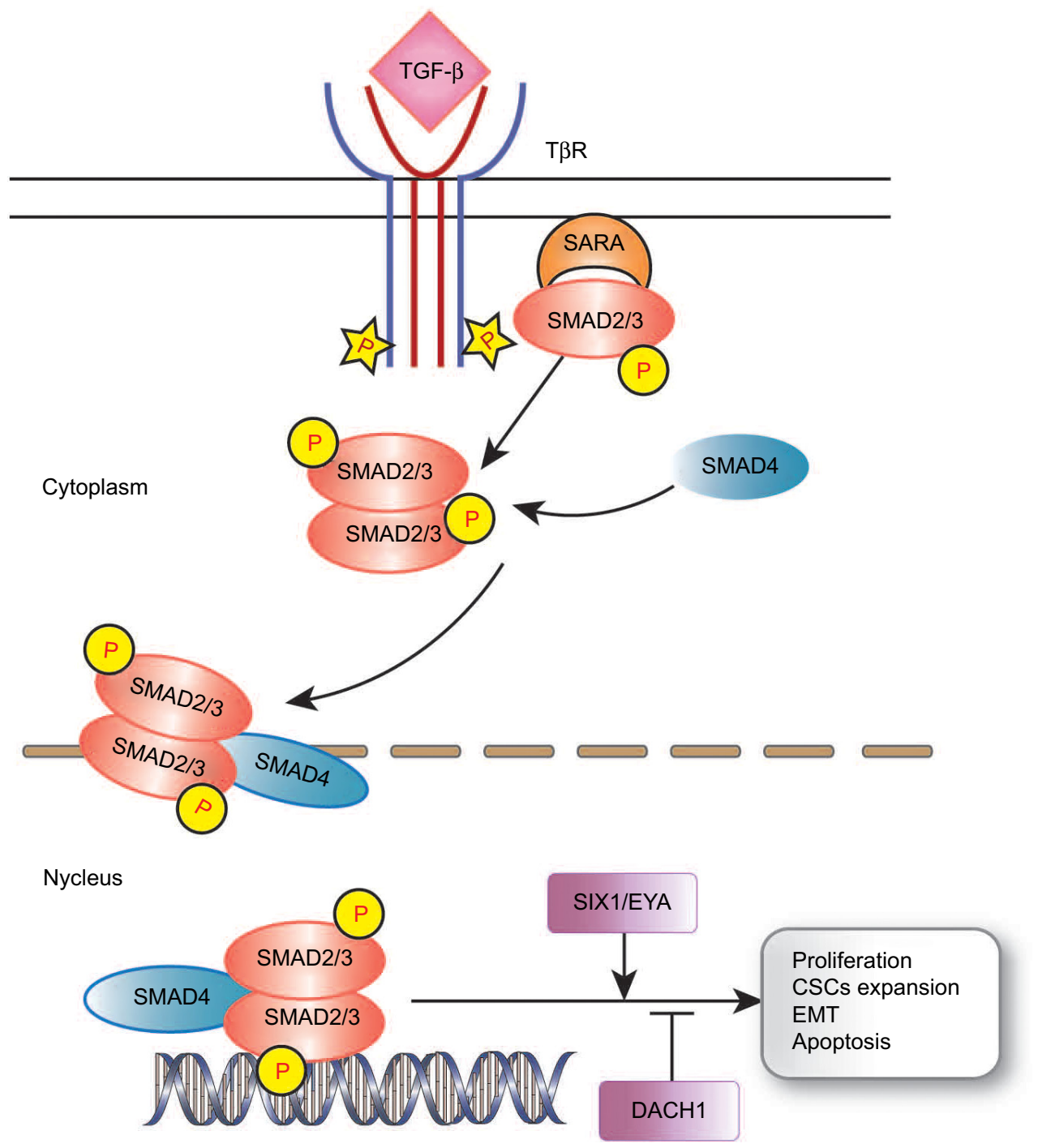

Figure 2 RDGN regulates TGF- $\beta$ signaling transduction in tumor cells.

Notes: TGF- $\beta$ binds to T $\beta$ R and activates SARA, which then recruits and activates intracellular SMAD2/3 protein. SMAD2/3 forms a heterodimer and enters into the nucleus with the assistance of SMAD4 protein. SMAD2/3/4 complex works as a transcription factor regulating the expression of targeted genes, which are associated with proliferation, CSCs' expansion, EMT, and apoptosis of various cancers. SIX/EYA compound acts as an activator of above process, while DACHI is an inhibitor of TGF- $\beta$ signaling.

Abbreviations: CSCs, cancer stem cells; EMT, epithelial-mesenchymal transition; RDGN, retinal determination gene network; SARA, SMAD anchor for receptor activation; T $\beta$ R, TGF- $\beta$ receptor. 
Table I Summarization of cytokines regulated by RDGN

\begin{tabular}{|c|c|c|c|c|}
\hline RDGN member & Regulated cytokines & Regulation direction & Tissue type & Reference \\
\hline \multirow[t]{20}{*}{$\mathrm{DACHI}$} & \multirow[t]{8}{*}{ TGF- $\beta$} & \multirow[t]{3}{*}{$\uparrow$} & Human adrenal tissue & 18 \\
\hline & & & Hepatocellular carcinoma & 35 \\
\hline & & & Esophageal cancer & 44 \\
\hline & & \multirow[t]{5}{*}{$\downarrow$} & Breast cancer & 39 \\
\hline & & & Colorectal cancer & 42 \\
\hline & & & Gastric cancer & 40 \\
\hline & & & Ovarian cancer & 43 \\
\hline & & & Gliomas & 72 \\
\hline & CXCLI2 & $\uparrow$ & Artery genesis & 51 \\
\hline & \multirow[t]{3}{*}{ CXCL8 } & \multirow[t]{3}{*}{$\downarrow$} & Breast cancer & 54 \\
\hline & & & Lung cancer & 55 \\
\hline & & & Prostate cancer & 56 \\
\hline & \multirow[t]{2}{*}{ CXCL5 } & $\downarrow$ & Lung cancer & 59 \\
\hline & & $\downarrow$ & Prostate cancer & 56 \\
\hline & \multirow[t]{2}{*}{$\mathrm{CXCLI} / 2$} & $\downarrow$ & Prostate cancer & 56 \\
\hline & & $\downarrow$ & Esophageal cancer & 57 \\
\hline & \multirow[t]{2}{*}{ IL-6 } & \multirow[t]{2}{*}{$\downarrow$} & Prostate cancer & 56 \\
\hline & & & Gliomas & 72 \\
\hline & \multirow[t]{2}{*}{ FGF } & $\downarrow$ & Gliomas & 72 \\
\hline & & $\downarrow$ & Prostate cancer & 56 \\
\hline \multirow[t]{6}{*}{ SIXI } & \multirow[t]{3}{*}{ TGF- $\beta$} & \multirow[t]{3}{*}{$\uparrow$} & Breast cancer & $45-47$ \\
\hline & & & Cervical cancer & 48 \\
\hline & & & Esophageal cancer & 49 \\
\hline & \multirow[t]{2}{*}{ VEGF-C } & \multirow[t]{2}{*}{$\uparrow$} & Breast cancer & 75 \\
\hline & & & Cervical cancer & 76 \\
\hline & TRAIL & $\downarrow$ & Ovarian cancer & 87 \\
\hline
\end{tabular}

Abbreviations: CXCL, C-X-C motif ligand; TRAIL, tumor necrosis factor-related apoptosis-inducing ligand.

blocked TGF- $\beta$ induction of activator protein-1 (AP-1) and Smad signaling. ${ }^{41} \mathrm{DACH} 1$ also retarded the invasive growth of colorectal tumor cells via interfering TGF- $\beta$-mediated EMT through perturbing the phosphorylation of Smad2. ${ }^{42}$ Sunde et al first proved that inhibitory signaling of TGF- $\beta$, including DACH1, BMP7, and EVI1, was upregulated in early-stage of ovarian cancer, which resulted in the resistance of ovarian cancer cells to TGF- $\beta$-induced antiproliferative and antiapoptotic effects. ${ }^{43}$ On the other hand, DACH1 was found to increase TGF- $\beta$ signaling in human adrenal tissue leading to the reduced aldosterone production. ${ }^{18}$ Besides, DACH1 controlled liver cell growth by reactivating TGF- $\beta$ signaling; thus, the loss of DACH1 expression in hepatocellular carcinoma impeded the antiproliferative function of TGF- $\beta$ and stimulated tumor growth. ${ }^{35}$ It was elucidated that DACH1 inhibited esophageal cancer growth by triggering and activating TGF- $\beta$ signaling both in vitro and in vivo (Table 1) ${ }^{44}$ Silencing of DACH1 caused by methylation in part contributed to the esophageal carcinogenesis by hampering TGF- $\beta{ }^{44}$
SIX1, known as a carcinogenic factor, facilitated the switch of TGF- $\beta$ from tumor suppressive signal to tumor promotional signal through upregulating TGF- $\beta$ type I receptor (T $\beta R I$ ) in breast cancer (Table 1) ${ }^{45}$ And SIX1-induced prometastatic phenotype of TGF- $\beta$ signaling further stimulated EMT and distant metastasis process in breast cancer transplant mouse model and also predicted poor survival of cancer patients. ${ }^{45,46}$ Furthermore, SIX1-induced nuclear Smad 3 recruitment resulted in the increased TGF- $\beta$ signaling in breast cancer. ${ }^{46}$ Besides promoting EMT, SIX1 induced the expansion of breast CSC population via activating TGF- $\beta$ and mitogen-activated protein kinase (MAPK) cascade pathways ${ }^{47}$ It was indicated that ectopic expression of SIX1regulated TGF- $\beta$-signaling network, including increased TGF- $\beta$ type II receptor (T $\beta$ RII), which then contributed to EMT and CSC properties of cervical cancer. ${ }^{48}$ The consistent result was obtained in a recent study that SIX1 led to the overexpression of TGF- $\beta$ and T $\beta$ RII and the acceleration of CSC self-renewal rate, inducing the progression and poor prognosis of esophageal squamous cell carcinoma. ${ }^{49}$ 
EYA coordinated with SIX1 to promote malignant phenotypes, including EMT, CSC expansion, and poor survival, through activating TGF- $\beta$ signaling in breast cancer..$^{38}$ Thus, EYA was recognized as a crucial activator of SIX1 and was required to mediate SIX1-induced TGF- $\beta$-dependent carcinogenic signal. Furthermore, deletion of Eya2 in MCF7 mammary carcinoma cells blocked SIX1-mediated tumor progression via TGF- $\beta$ signaling. ${ }^{38}$

\section{C-X-C motif ligand (CXCL) family}

CXCL family is well known as a prototypical chemokine playing roles in inflammation, immune response, and cancer process. Inflammatory responses to CXCL factors in the tumor microenvironment are responsible to facilitate tumor growth, progression, and immunosuppression.$^{50}$ Regulation of CXCL family by RDGN is summarized in Table 1.

A recent study showed that CXCL12 and its receptor CXCR4 were potential targets of DACH1 in the process of arteries development. DACH1-stimulated CXCL12 expression promoted the migration of arterial endothelial cells against blood flow, which contributed to vascular development in vitro. ${ }^{51-53}$ Whereas, knock down of CXCR4-CXCL12 signaling could impair DACH1-induced endothelial expression and migration both in vitro and in vivo (Table 1). ${ }^{51}$ Additionally, DACH1 repressed CXCL8 expression through Dach and Sno/Ski homolog domain (DS domain) binding to CXCL8 promoter in a dose-dependent manner, which caused to the reduced migration of cancer cells in vitro and distant lung metastasis in vivo. ${ }^{54,55}$ In the context of prostate cancer, enhanced CXCL8 signaling contributed to castrateresistant prostate cancer (CRPC) development and promoted the invasion of cancer cells, whereas endogenous DACH1 inhibited the transcription and expression of CXCL8 by interacting with CXCL8 promoters, which in turn restrained prostate epithelial cell growth and migration (Table 1). ${ }^{56}$ According to microarray gene expression profile, CXCL1 and CXCL2 were also significantly decreased in PC-3 cells stably expressing DACH1 (Table 1).$^{56}$ Secretion of growth-regulated alpha protein (GRO- $\alpha$ and CXCL1) and macrophage inflammatory protein 1-alpha (MIP-1 $\alpha$ ) was also reported to be inhibited by DACH1 in human breast cancer cell line MDA-MB-231 and prostate cancer cell. ${ }^{54}$ It has been observed that metformin inhibited CXCL1 protein abundance by inducing the expression of DACH1 in cultured esophageal cancer cell and xenograft tumor tissues. Moreover, knockdown of DACH1 expression blocked the effect of metformin on myeloid-derived suppressor cells' (MDSCs) chemotaxis (Table 1). ${ }^{57}$ However, it is hard to separate direct antitumor effect of DACH1 on tumor cell to indirect tumor inhibition from immune modification by this in vivo xenograft model.

A recent study showed that serum CXCL5 concentration was significantly higher in non-small-cell lung cancer (NSCLC) compared with that in healthy volunteers. Moreover, cancer tissue CXCL5 mRNA transcription and protein expression correlated with tumor stage and predicted poor overall survival in adenocarcinoma (ADC) patients. ${ }^{58} \mathrm{CXCL} 5$ was also identified as a downstream target of DACH1 in lung ADC. DACH1 inversely correlated with the CXCL5 expression and hampered the CXCL5-stimulated invasion and migration of ADC cells (Table 1). ${ }^{59}$

Most CXCL family members are transcriptionally activated by MAPK signaling and NF- $\kappa$ B pathway. ${ }^{50,54,55}$ DACH1 was known to block the activity of MAPK signaling and NF-אB pathway induced by tumor necrosis factor $\alpha$ (TNF- $\alpha$ ), TPA, and serum stimulation, ${ }^{9,55}$ which may explain the inhibitory effect of DACH1 on a panel of cytokines at molecular level. DACH1 inhibits cancer cell proliferation and invasion through reducing cyclin D1, stemness, and EMT. The paracrine signaling not only stimulates tumor cell proliferation but also reprograms tumor environment and modulates tumor immune response. Therefore, the effects of DACH1 on intracellular and extracellular signals contribute to the in vivo tumor suppressor function. While recovering the nuclear protein DACH1 is still a technical challenge at this moment, blocking the hyperactivity of cytokines by neutralized antibody or specific inhibitor in DACH1-defected tumors may be feasible. Overall, DACH1 plays a vital role in the processes of organ development and tumor progression via regulating CXCL signaling pathways in a cell- and tissue-dependent manner. Nevertheless, SIX and EYA have not been found to interact with CXCL family.

\section{IL-I and IL-6}

IL-1 is a pleiotropic cytokine involved in various immune responses, inflammatory processes, and hematopoiesis. It is normally produced by monocytes and macrophages. Recent study showed that tumor-derived IL- $1 \alpha$, acting on infiltrating myeloid cells, induced the expression of a critical tumor survival factor TSLP and promoted breast cancer metastasis. ${ }^{60}$ Ecotopic expression of DACH1 significantly inhibited the secretion of IL- $1 \alpha$ in human breast cancer cell line MDA-MB-231 and blocked breast cancer cell metastasis to lung. ${ }^{54}$

IL-6, another proinflammatory cytokine, participated in various physiological and pathological processes by interacting with diverse intracellular signaling pathways. ${ }^{61}$ Previous studies showed that IL-6 was elevated in various cancers. ${ }^{62,63}$ 
Aberrant expression of IL-6 is correlated with high tumor burden, such as advanced stage and poor prognosis. Using Dach $1^{\mathrm{f} / \mathrm{fl}} /$ Probasin-Cre bitransgenic mice, Chen et al showed that endogenous Dach1 served as a key endogenous restraint to prostate epithelial cell growth and restrained migration via IL-6 signaling. Further mechanism study identified that DS domain of DACH1 was required for the repression of IL-6 promoter activity (Table 1$).^{56}$

\section{Fibroblast growth factor 2 (FGF2)}

FGF2, also named basic fibroblast growth factor (bFGF) and FGF- $\beta$, is a growth factor and signaling molecule involving a wide range of biological processes, including cell proliferation, morphogenesis, differentiation, angiogenesis, tumor growth, and invasion. ${ }^{64-67}$ It is worth mentioning that FGF2 is an indispensable factor in the maintenance of self-renewal of glioma stem cells and promotes tumor-initiating spheroid formation of glioma cells. ${ }^{68,69}$ And overexpression of FGF2 was frequently detected in high-grade gliomas and involved in glioma progression..$^{70,71}$ Watanabe et al $^{72}$ conducted gene expression analysis and chromatin immunoprecipitation assay to prove that FGF2 is transcriptionally repressed by DACH1 (Table 1). FGF2 enhanced the tumorigenicity characteristics of glioma stem cells in vitro and in vivo, while stable expression of DACH1 undermined this function. At the meantime, exogenous bFGF rescues stemness and tumorigenicity of the U87 cells with high expression of DACH1, suggesting that loss of DACH1 increases the number of CSC through transcriptional activation of bFGF. ${ }^{72}$ Intriguingly, DACH1 was upregulated by growth factor signaling, such as FGF2, in the process of limb skeletal development and acted as an intermediary in the FGF signaling pathway to regulate cell proliferation or differentiation. ${ }^{13}$

\section{Vascular endothelial growth factor (VEGF)}

VEGF C (VEGF-C) is a member of the platelet-derived growth factor (PDGF)/VEGF family. Lymphangiogenesis is a major function of VEGF-C. It promotes survival, proliferation, and migration of lymphatic endothelial cells, inducing the establishment of lymphatic vessels, through binding to VEGF receptor 3. ${ }^{73,74}$ All these characteristics of VEGF-C provided a favorite environment for the dissemination of cancer cells.

SIX1 induced lymphangiogenesis and lymphatic metastasis of breast cancer and cervical cancer partially through promoting the transcription and expression of VEGF-C both in cell lines and xenograft mouse model (Table 1). ${ }^{75,76}$
Therefore, it was elucidated that SIX1/VEGF-C axis played a vital role in the lymphatic dissemination of breast cancer cells. Interestingly, recovery of VEGF-C in SIX1 knockdown breast cancer cells promoted lymphatic metastasis but not distant metastases to the lungs. ${ }^{75}$ Additionally, TGF- $\beta$ was recognized as an intermediate in SIX-stimulated VEGF-C expression. SIX1 augmented the TGF- $\beta$-activated SMAD2/3 and then assisted with the SMAD pathway to modulate VEGF-C expression. ${ }^{76}$ Although TGF- $\beta$ inhibited lymphangiogenesis by limiting tube formation of lymphatic endothelial cells, the increased VEGF-C could counteract the inhibitory effect of TGF- $\beta$ and promoted the migration of lymphatic endothelial cells directly. ${ }^{76}$ Another study showed that nuclear DACH1 repressed the microvascular density (MVD), but whether this effect relies on the inhibition of VEGF is not known. ${ }^{77}$

\section{Other factors}

TNF-related apoptosis-inducing ligand (TRAIL), belonging to the TNF cytokine family, is a protein triggering the process of apoptosis. ${ }^{78,79}$ Generally, TRAIL secreted by normal tissue cells induces apoptosis primarily in tumor cells via interacting with specific death receptors. ${ }^{78}$ For instance, TRAIL triggered extrinsic apoptotic pathway by activating the death receptor 4 (DR4) and death receptor 5 (DR5) expressed on the cell membrane, which then recruited intracellular death adaptor molecule to form the death-inducing signaling complex and resulted in cell death. ${ }^{80-84}$ By starting the apoptosis program, TRAIL was involved in tumor suppression, such as enhancing innate immunosurveillance and inhibiting tumorigenesis and tumor progression. ${ }^{85}$ Moreover, TRAIL was proved to have cytotoxic activity against ovarian carcinoma cells both in vitro and in vivo. ${ }^{86}$

High mRNA expression of $S I X 1$ was found to be related with late stage and worse prognosis of ovarian carcinoma (Table 1). ${ }^{87}$ In terms of mechanism, SIX1 prevented ovarian carcinoma cells from TRAIL-mediated cell death and contributed to the tumorigenesis and distant metastasis of ovarian carcinoma via conferring resistance to apoptosisrelated process of tumor cells. ${ }^{87}$ Inhibition of TRAIL pathway by SIX1 impaired the immunosurveillance activity of natural killer cells against tumor cells and led to poor survival in patients.

Receptor activator of nuclear factor kappa-B ligand (RANKL) is a member of the TNF superfamily and a ligand for the receptor activator of nuclear factor kappa-B (RANK). RANKL controls cell proliferation, apoptosis, and immune functions. ${ }^{88} \mathrm{DACH} 1$ was found to repress FGF2-enhanced 
RANKL expression in stromal/preosteoblast cells in associated with $\mathrm{NCoR} .{ }^{89}$

\section{Conclusion}

RDGN pathway acts as a tumor regulator mainly through interfering the transcription of targeted genes. It is acknowledged that most of cytokine pathways can promote the proliferation, tumorigenesis, angiogenesis, and metastasis of tumor cells. Specifically, cytokine is a critical mediator in the regulatory function of RDGN in tumor initiation and progression. Thus, DACH1, as a tumor suppressor, retards the tumor growth through blocking cytokine signaling, such as TGF- $\beta$, CXCL1, CXCL5, CXCL8, and FGF. While another member of RDGN, SIX1 facilitates tumor progression by stimulating cytokines' secretion and activating the pathways triggered by cytokine factors. EYA-SIX forms a transcriptional complex to activate TGF- $\beta$ signaling and induce the EMT of cancers. Overall, there is a tight interaction between RDGN and cytokine factors in tumorigenesis and progression and its effect is cell type dependent.

\section{Acknowledgments}

This work was supported by the National Natural Science Foundation of China (Nos 81572608, 81874120, and 81172422 ) and Wuhan Science and Technology Bureau (No 2017060201010170).

\section{Author contributions}

$\mathrm{XZ}$ and QL collected the paper and wrote the manuscript. MY and SQ discussed and revised the manuscript. KW designed the manuscript. All authors contributed toward data analysis, drafting and revising the paper and agree to be accountable for all aspects of the work.

\section{Disclosure}

The authors report no conflicts of interest in this work.

\section{References}

1. Dinarello CA. Proinflammatory cytokines. Chest. 2000;118(2): 503-508.

2. Saito S. Cytokine cross-talk between mother and the embryo/placenta. $J$ Reprod Immunol. 2001;52(1-2):15-33.

3. Chen HF, Shew JY, Ho HN, Hsu WL, Yang YS. Expression of leukemia inhibitory factor and its receptor in preimplantation embryos. Fertil Steril. 1999;72(4):713-719.

4. Jemc J, Rebay I. Targeting Drosophila eye development. Genome Biol. 2006;7(7):226.

5. Pignoni F, Hu B, Zavitz KH, Xiao J, Garrity PA, Zipursky SL. The eye-specification proteins So and Eya form a complex and regulate multiple steps in Drosophila eye development. Cell. 1997;91(7): 881-891.

6. Miller SJ, Lan ZD, Hardiman A, et al. Inhibition of Eyes Absent Homolog 4 expression induces malignant peripheral nerve sheath tumor necrosis. Oncogene. 2010;29(3):368-379.
7. Liu $\mathrm{Y}$, Kong $\mathrm{D}, \mathrm{Wu} \mathrm{H}$, et al. Interplay of retinal determination gene network with TGF- $\beta$ signaling pathway in epithelial-mesenchymal transition. Stem Cell Investig. 2015;2:12.

8. Kumar JP. The molecular circuitry governing retinal determination. Biochim Biophys Acta. 2009;1789(4):306-314.

9. Wu K, Liu M, Li A, et al. Cell fate determination factor DACH1 inhibits c-Jun-induced contact-independent growth. Mol Biol Cell. 2007; 18(3):755-767.

10. Popov VM, Zhou J, Shirley LA, et al. The cell fate determination factor DACH1 is expressed in estrogen receptor-alpha-positive breast cancer and represses estrogen receptor-alpha signaling. Cancer Res. 2009;69(14):5752-5760.

11. Li X, Oghi KA, Zhang J, et al. Eya protein phosphatase activity regulates Six1-Dach-Eya transcriptional effects in mammalian organogenesis. Nature. 2003;426(6964):247-254.

12. Ikeda K, Watanabe Y, Ohto H, Kawakami K. Molecular interaction and synergistic activation of a promoter by Six, Eya, and Dach proteins mediated through CREB binding protein. Mol Cell Biol. 2002;22(19): 6759-6766.

13. Horner A, Shum L, Ayres JA, Nonaka K, Nuckolls GH. Fibroblast growth factor signaling regulates Dach1 expression during skeletal development. Dev Dyn. 2002;225(1):35-45.

14. Kalousova A, Mavropoulos A, Adams BA, et al. Dachshund homologues play a conserved role in islet cell development. Dev Biol. 2010;348(2):143-152.

15. Schild R, Knüppel T, Konrad M, et al. Double homozygous missense mutations in DACH1 and BMP4 in a patient with bilateral cystic renal dysplasia. Nephrol Dial Transplant. 2013;28(1):227-232.

16. Köttgen A, Pattaro C, Böger CA, et al. New loci associated with kidney function and chronic kidney disease. Nat Genet. 2010;42(5): 376-384.

17. Ma RC, Lee HM, Lam VK, et al. Familial young-onset diabetes, prediabetes and cardiovascular disease are associated with genetic variants of DACH1 in Chinese. PLoS One. 2014;9(1):e84770.

18. Zhou J, Shaikh LH, Neogi SG, et al. DACH1, a zona glomerulosa selective gene in the human adrenal, activates transforming growth factor- $\beta$ signaling and suppresses aldosterone secretion. Hypertension. 2015;65(5):1103-1110.

19. Patrick AN, Cabrera JH, Smith AL, Chen XS, Ford HL, Zhao R. Structure-function analyses of the human SIX1-EYA2 complex reveal insights into metastasis and BOR syndrome. Nat Struct Mol Biol. 2013;20(4):447-453.

20. Abdelhak S, Kalatzis V, Heilig R, et al. A human homologue of the Drosophila eyes absent gene underlies branchio-oto-renal (BOR) syndrome and identifies a novel gene family. Nat Genet. 1997;15(2):157-164

21. Ruf RG, Xu PX, Silvius D, et al. SIX1 mutations cause branchio-otorenal syndrome by disruption of EYA1-SIX1-DNA complexes. Proc Natl Acad Sci U S A. 2004;101(21):8090-8095.

22. Wu K, Li Z, Cai S, et al. EYA1 phosphatase function is essential to drive breast cancer cell proliferation through cyclin D1. Cancer Res. 2013;73(14):4488-4499.

23. Yuan B, Cheng L, Chiang HC, et al. A phosphotyrosine switch determines the antitumor activity of ER $\beta$. J Clin Invest. 2014;124(8):3378-3390.

24. Liu Y, Han N, Zhou S, et al. The DACH/EYA/SIX gene network and its role in tumor initiation and progression. Int J Cancer. 2016;138(5): $1067-1075$

25. Wu W, Ren Z, Li P, et al. Six 1: a critical transcription factor in tumorigenesis. Int J Cancer. 2015;136(6):1245-1253

26. Zou D, Silvius D, Fritzsch B, Xu PX. Eya1 and Six1 are essential for early steps of sensory neurogenesis in mammalian cranial placodes. Development. 2004;131(22):5561-5572.

27. Hu S, Mamedova A, Hegde RS. DNA-binding and regulation mechanisms of the SIX family of retinal determination proteins. Biochemistry. 2008;47(11):3586-3594.

28. Chu Q, Han N, Yuan X, et al. DACH1 inhibits cyclin D1 expression, cellular proliferation and tumor growth of renal cancer cells. J Hematol Oncol. 2014;7:73. 
29. Kong D, Liu Y, Liu Q, et al. The retinal determination gene network: from developmental regulator to cancer therapeutic target. Oncotarget. 2016;7(31):50755-50765.

30. Massagué J. TGFbeta in Cancer. Cell. 2008;134(2):215-230.

31. Siegel PM, Massagué J. Cytostatic and apoptotic actions of TGF-beta in homeostasis and cancer. Nat Rev Cancer. 2003;3(11):807-821.

32. Akhurst RJ, Hata A. Targeting the TGF $\beta$ signalling pathway in disease. Nat Rev Drug Discov. 2012;11(10):790-811.

33. Lebrun JJ. The Dual Role of TGF $\beta$ in Human Cancer: From Tumor Suppression to Cancer Metastasis. ISRN Mol Biol. 2012;2012:381428.

34. Chen CR, Kang Y, Massagué J. Defective repression of c-myc in breast cancer cells: A loss at the core of the transforming growth factor beta growth arrest program. Proc Natl Acad Sci U S A. 2001;98(3): 992-999.

35. Zhu H, Wu K, Yan W, et al. Epigenetic silencing of DACH1 induces loss of transforming growth factor- $\beta 1$ antiproliferative response in human hepatocellular carcinoma. Hepatology. 2013;58(6):2012-2022.

36. Saitoh M, Miyazawa K. Transcriptional and post-transcriptional regulation in TGF- $\beta$-mediated epithelial-mesenchymal transition. J Biochem. 2012;151(6):563-571.

37. Hasegawa T, Yashiro M, Nishii T, et al. Cancer-associated fibroblasts might sustain the stemness of scirrhous gastric cancer cells via transforming growth factor- $\beta$ signaling. Int $J$ Cancer. 2014;134(8):1785-1795.

38. Farabaugh SM, Micalizzi DS, Jedlicka P, Zhao R, Ford HL. Eya2 is required to mediate the pro-metastatic functions of Six 1 via the induction of TGF- $\beta$ signaling, epithelial-mesenchymal transition, and cancer stem cell properties. Oncogene. 2012;31(5):552-562.

39. Wu K, Yang Y, Wang C, et al. DACH1 inhibits transforming growth factor-beta signaling through binding Smad4. J Biol Chem. 2003;278(51):51673-51684.

40. Yan W, Wu K, Herman JG, et al. Epigenetic silencing of DACH1 induces the invasion and metastasis of gastric cancer by activating TGF- $\beta$ signalling. J Cell Mol Med. 2014;18(12):2499-2511.

41. Wilson JJ, Malakhova M, Zhang R, Joachimiak A, Hegde RS. Crystal structure of the dachshund homology domain of human SKI. Structure. 2004;12(5):785-792.

42. Wang P. Suppression of DACH1 promotes migration and invasion of colorectal cancer via activating TGF- $\beta$-mediated epithelial-mesenchymal transition. Biochem Biophys Res Commun. 2015;460(2):314-319.

43. Sunde JS, Donninger H, Wu K, et al. Expression profiling identifies altered expression of genes that contribute to the inhibition of transforming growth factor-beta signaling in ovarian cancer. Cancer Res. 2006;66(17):8404-8412.

44. Wu L, Herman JG, Brock MV, et al. Silencing DACH1 promotes esophageal cancer growth by inhibiting TGF- $\beta$ signaling. PLoS One. 2014; 9(4):e95509.

45. Micalizzi DS, Wang CA, Farabaugh SM, Schiemann WP, Ford HL. Homeoprotein Six1 increases TGF-beta type I receptor and converts TGF-beta signaling from suppressive to supportive for tumor growth. Cancer Res. 2010;70(24):10371-10380.

46. Micalizzi DS, Christensen KL, Jedlicka P, et al. The Six1 homeoprotein induces human mammary carcinoma cells to undergo epithelialmesenchymal transition and metastasis in mice through increasing TGF-beta signaling. J Clin Invest. 2009;119(9):2678-2690.

47. Iwanaga R, Wang CA, Micalizzi DS, et al. Expression of Six1 in luminal breast cancers predicts poor prognosis and promotes increases in tumor initiating cells by activation of extracellular signal-regulated kinase and transforming growth factor-beta signaling pathways. Breast Cancer Res. 2012;14(4):R100.

48. Xu H, Zhang Y, Altomare D, et al. Six1 promotes epithelial-mesenchymal transition and malignant conversion in human papillomavirus type 16-immortalized human keratinocytes. Carcinogenesis. 2014;35(6):1379-1388.

49. Nishimura T, Tamaoki M, Komatsuzaki R, et al. SIX1 maintains tumor basal cells via transforming growth factor- $\beta$ pathway and associates with poor prognosis in esophageal cancer. Cancer Sci. 2017;108(2):216-225.
50. Liu Q, Li A, Tian Y, et al. The CXCL8-CXCR1/2 pathways in cancer. Cytokine Growth Factor Rev. 2016;31:61-71.

51. Chang AH, Raftrey BC, D'Amato G, et al. DACH1 stimulates shear stress-guided endothelial cell migration and coronary artery growth through the CXCL12-CXCR4 signaling axis. Genes Dev. 2017;31(13):1308-1324.

52. Strasser GA, Kaminker JS, Tessier-Lavigne M. Microarray analysis of retinal endothelial tip cells identifies CXCR4 as a mediator of tip cell morphology and branching. Blood. 2010;115(24):5102-5110.

53. Bussmann J, Wolfe SA, Siekmann AF. Arterial-venous network formation during brain vascularization involves hemodynamic regulation of chemokine signaling. Development. 2011;138(9):1717-1726.

54. Wu K, Katiyar S, Li A, et al. Dachshund inhibits oncogene-induced breast cancer cellular migration and invasion through suppression of interleukin-8. Proc Natl Acad Sci U S A. 2008;105(19):6924-6929.

55. Liu Q, Li A, Yu S, et al. DACH1 antagonizes CXCL8 to repress tumorigenesis of lung adenocarcinoma and improve prognosis. $J$ Hematol Oncol. 2018;11(1):53.

56. Chen $\mathrm{K}, \mathrm{Wu} \mathrm{K}$, Jiao X, et al. The endogenous cell-fate factor dachshund restrains prostate epithelial cell migration via repression of cytokine secretion via a cxcl signaling module. Cancer Res. 2015; 75(10):1992-2004.

57. Qin G, Lian J, Huang L, et al. Metformin blocks myeloid-derived suppressor cell accumulation through AMPK-DACH1-CXCL1 axis. Oncoimmunology. 2018;7(7):e1442167.

58. Wu K, Yu S, Liu Q, Bai X, Zheng X, Wu K. The clinical significance of CXCL5 in non-small cell lung cancer. Onco Targets Ther. 2017; 10:5561-5573.

59. Han N, Yuan X, Wu H, et al. DACH1 inhibits lung adenocarcinoma invasion and tumor growth by repressing CXCL5 signaling. Oncotarget. 2015;6(8):5877-5888.

60. Kuan EL, Ziegler SF. A tumor-myeloid cell axis, mediated via the cytokines IL- $1 \alpha$ and TSLP, promotes the progression of breast cancer. Nat Immunol. 2018;19(4):366-374.

61. Liu Q, Yu S, Li A, Xu H, Han X, Wu K. Targeting interlukin-6 to relieve immunosuppression in tumor microenvironment. Tumour Biol. 2017;39(6):1010428317712445.

62. Guo Y, Xu F, Lu T, Duan Z, Zhang Z. Interleukin-6 signaling pathway in targeted therapy for cancer. Cancer Treat Rev. 2012;38(7): 904-910.

63. Liu G, Zhang J, Frey L, et al. Prostate-specific IL-6 transgene autonomously induce prostate neoplasm through amplifying inflammation in the prostate and peri-prostatic adipose tissue. J Hematol Oncol. 2017; 10(1): 14 .

64. Ornitz DM. Regulation of chondrocyte growth and differentiation by fibroblast growth factor receptor 3. Novartis Found Symp. 2001;232: 63-76; discussion 76-80, 272-282.

65. Itoh N, Ornitz DM. Evolution of the Fgf and Fgfr gene families. Trends Genet. 2004;20(11):563-569.

66. Angelin B, Larsson TE, Rudling M. Circulating fibroblast growth factors as metabolic regulators - a critical appraisal. Cell Metab. 2012;16(6):693-705.

67. Bikfalvi A, Klein S, Pintucci G, Rifkin DB. Biological roles of fibroblast growth factor-2. Endocr Rev. 1997;18(1):26-45.

68. Galli R, Binda E, Orfanelli U, et al. Isolation and characterization of tumorigenic, stem-like neural precursors from human glioblastoma. Cancer Res. 2004;64(19):7011-7021.

69. Singh SK, Clarke ID, Terasaki M, et al. Identification of a cancer stem cell in human brain tumors. Cancer Res. 2003;63(18):5821-5828.

70. Takahashi JA, Fukumoto M, Igarashi K, Oda Y, Kikuchi H, Hatanaka M. Correlation of basic fibroblast growth factor expression levels with the degree of malignancy and vascularity in human gliomas. J Neurosurg. 1992;76(5):792-798.

71. Yi W, Chen J, Golwa FH, Xue D. Basic fibroblast growth factor and fibroblast growth factor receptor-i in human meningiomas. J Huazhong Univ Sci Technolog Med Sci. 2005;25(1):75-77. 
72. Watanabe A, Ogiwara H, Ehata S, et al. Homozygously deleted gene DACH1 regulates tumor-initiating activity of glioma cells. Proc Natl Acad Sci U S A. 2011;108(30):12384-12389.

73. Oh SJ, Jeltsch MM, Birkenhäger R, et al. VEGF and VEGF-C: specific induction of angiogenesis and lymphangiogenesis in the differentiated avian chorioallantoic membrane. Dev Biol. 1997;188(1): 96-109.

74. Jeltsch M, Kaipainen A, Joukov V, et al. Hyperplasia of lymphatic vessels in VEGF-C transgenic mice. Science. 1997;276(5317):1423-1425.

75. Wang CA, Jedlicka P, Patrick AN, et al. SIX1 induces lymphangiogenesis and metastasis via upregulation of VEGF-C in mouse models of breast cancer. J Clin Invest. 2012;122(5):1895-1906.

76. Liu D, Li L, Zhang XX, et al. SIX1 promotes tumor lymphangiogenesis by coordinating TGF $\beta$ signals that increase expression of VEGF-C. Cancer Res. 2014;74(19):5597-5607.

77. Ren P, Gong MZ, Wang ZY, et al. DACH1 Expresison in Osteosarcoma and Its Relationship with Proliferation and Angiogenesis. Indian J Surg 2015;77(3):200-205.

78. Wiley SR, Schooley K, Smolak PJ, et al. Identification and characterization of a new member of the TNF family that induces apoptosis. Immunity. 1995;3(6):673-682.

79. Pitti RM, Marsters SA, Ruppert S, Donahue CJ, Moore A, Ashkenazi A. Induction of apoptosis by Apo-2 ligand, a new member of the tumor necrosis factor cytokine family. J Biol Chem. 1996;271(22): 12687-12690

80. Ozören N, El-Deiry WS. Cell surface Death Receptor signaling in normal and cancer cells. Semin Cancer Biol. 2003;13(2):135-147.

81. Nagane M, Huang HJ, Cavenee WK. The potential of TRAIL for cancer chemotherapy. Apoptosis. 2001;6(3):191-197.
82. Thomas LR, Henson A, Reed JC, Salsbury FR, Thorburn A. Direct binding of Fas-associated death domain (FADD) to the tumor necrosis factorrelated apoptosis-inducing ligand receptor DR5 is regulated by the death effector domain of FADD. J Biol Chem. 2004;279(31):32780-32785.

83. Thomas LR, Johnson RL, Reed JC, Thorburn A. The C-terminal tails of tumor necrosis factor-related apoptosis-inducing ligand (TRAIL) and Fas receptors have opposing functions in Fas-associated death domain (FADD) recruitment and can regulate agonist-specific mechanisms of receptor activation. J Biol Chem. 2004;279(50):52479-52486.

84. Rowinsky EK. Targeted induction of apoptosis in cancer management: the emerging role of tumor necrosis factor-related apoptosisinducing ligand receptor activating agents. J Clin Oncol. 2005;23(36): 9394-9407.

85. Cretney E, Takeda K, Yagita H, Glaccum M, Peschon JJ, Smyth MJ. Increased susceptibility to tumor initiation and metastasis in TNF-related apoptosis-inducing ligand-deficient mice. J Immunol. 2002;168(3): 1356-1361.

86. Liu P, Mao H, Hou P. Synergistic antitumor effect of tumor necrosis factor-related apoptosis-inducing ligand combined with cisplatin in ovarian carcinoma cell lines in vitro and in vivo. Int J Gynecol Cancer. 2006;16(2):538-548.

87. Behbakht K, Qamar L, Aldridge CS, et al. Six1 overexpression in ovarian carcinoma causes resistance to TRAIL-mediated apoptosis and is associated with poor survival. Cancer Res. 2007;67(7):3036-3042.

88. González-Suárez E, Sanz-Moreno A. RANK as a therapeutic target in cancer. FEBS J. 2016;283(11):2018-2033.

89. Sundaram K, Mani SK, Kitatani K, Wu K, Pestell RG, Reddy SV. DACH1 negatively regulates the human RANK ligand gene expression in stromal/preosteoblast cells. J Cell Biochem. 2008;103(6):1747-1759.
OncoTargets and Therapy

\section{Publish your work in this journal}

OncoTargets and Therapy is an international, peer-reviewed, open access journal focusing on the pathological basis of all cancers, potential targets for therapy and treatment protocols employed to improve the management of cancer patients. The journal also focuses on the impact of management programs and new therapeutic agents and protocols on

\section{Dovepress}

patient perspectives such as quality of life, adherence and satisfaction. The manuscript management system is completely online and includes a very quick and fair peer-review system, which is all easy to use. Visit http://www.dovepress.com/testimonials.php to read real quotes from published authors. 